\title{
Чтобы быстрее двигаться вперед, компаниям нужны не отдельные измерительные приборы, а законченные решения
}

Рассказывает президент Группы коммуникационных решений компании Keysight Technologies C. Данасекаран

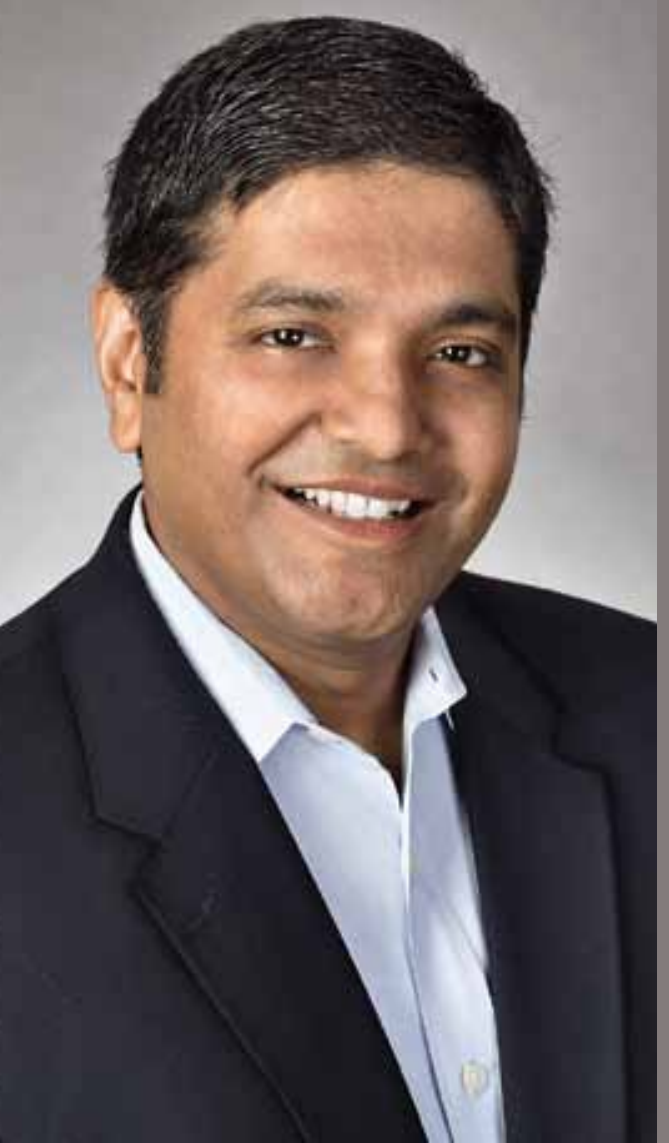

В начале апреля текущего года между Ооо «Кейсайт Текнолоджиз» - российским подразделением компании Keysight Technologies, и АО «Технопарк Санкт-Петербурга" был подписан меморандум о сотрудничестве. Среди направлений, обладающих высоким потенциалом на российском рынке, Keysight Technologies видит развитие технологий телекоммуникационных сетей нового поколения 5С - одной из областей, в которых у данной компании есть самые передовые решения, способные помочь в создании мощных конкурентоспособных систем.

О том, какие особенности связаны с технологиями 5С, в частности, в области средств контроля и измерений, в каком состоянии находится сейчас процесс стандартизации в отношении этих сетей, как меняются потребности компаний, использующих контрольно-измерительное оборудование, и как отвечает всем этим новым требованиям Keysight Technologies, нам рассказал президент Группы коммуникационных решений компании Сатиш Данасекаран (Satish Dhanasekaran).
Господин Данасекаран, 11 апреля был подписан меморандум о сотрудничестве между ООО "Кейсайт Текнолоджиз" и АО "Технопарк Санкт-Петербурга". На какой области радиоэлектроники планируется сосредоточить взаимодействие вашей компании и технопарка?

Наша компания занимает лидирующие позиции в сфере средств контроля и измерений для создания электронных устройств и систем в различных областях. И наше сотрудничество с Технопарком Санкт-Петербурга не будет ограничено каким-то одним направлением. Подписание данного меморандума открывает новые возможности для реализации проектов инновационных предприятий и вузов СанктПетербурга в разных секторах радиоэлектронной отрасли. Мы надеемся, что благодаря этому сотрудничеству предприятия получат доступ к нашим инновационным решениям в дополнение к использованию ими имеющихся технологических возможностей технопарка, что поможет им в создании конкурентоспособной продукции различного назначения, а также в том, 
чтобы выводить ее на рынок в возможно короткие сроки.

Но если говорить о конкретных направлениях, я особо остановился бы на такой активно развивающейся области, как телекоммуникационные сети пятого поколения. Во многих странах сейчас существует понимание, что построение сетей 5 С создаст почву для развития огромного количества инноваций и окажет реальное содействие развитию экономики государства. Поэтому в этом направлении существует активное движение и в США, и в Китае, и во многих странах Евросоюза. Мы видим большое внимание к данным технологиям и в России и, имея значительные компетенции в этой области, можем оказать существенную помощь российским предприятиям-разработчикам, интеграторам, операторам и другим компаниям, работающим в направлении развертывания и использования сетей 5G.

Сейчас по всему миру создаются новые технологии, многие из которых находятся еще на самой ранней, "инкубационной" стадии. И мы видим свою миссию в том, чтобы ускорять инновационные процессы, которые улучшают коммуникации по всей нашей планете и делают их более безопасными.

\section{Это касается инфраструктурных средств или} также и абонентских устройств?

Это касается и того и другого. Вообще, в отношении технологий 5 С можно и нужно говорить о сквозных решениях, о характеристиках всей коммуникационной системы, начиная от подключенного к сети устройства, которое может быть смартфоном, ІоТ-устройством или, например, автомобилем, и заканчивая облаком. Более того, эта цепочка включает в себя не только аппаратные, но и программные средства, потому что сейчас всё в большей мере растет роль виртуализации, обработки данных с помощью ПО в дата-центрах.

Поэтому компаниям, работающим в этой области, как и во многих других перспективных направлениях, уже недостаточно отдельных инструментов, позволяющих выполнить узкую задачу. Им необходимы комплексные решения, которые позволят не только измерить те или иные характеристики, но увидеть, какие параметры достигаются их изделием в рамках общей системы.

И чтобы обеспечить такие потребности наших заказчиков, компания меняется. Мы многие годы работали как поставщик контрольно-измерительных приборов, но сейчас мы вышли за эти рамки и предоставляем нашим клиентам законченные решения под ключ, тем самым действительно помогая им решать многие стоящие перед ними задачи быстрее.

Конечно, это не означает, что контрольноизмерительному оборудованию мы уделяем меньше внимания. Мы предлагаем широкий спектр приборов, который постоянно обновляется и дополняется. Но помимо этого, например, у нас есть решения для эмуляции как сетей, так и устройств 5G. И это позволяет заказчику определить характеристики всей системы. Само собой, вы можете измерить параметры рч- и цифровых сигналов, но также с помощью наших решений вы можете выполнить эмуляцию всего набора протоколов 5С и всего ПО.

\section{Мы многие годы работали как поставщик контрольно- измерительных приборов, но сейчас мы вышии за эти рамки и предоставляем нашим кииентам законченные решения под киюч, тем самым действительно помогая им решать многие стоящие перед ними задачи бolcmpee}

У нас есть все необходимые компоненты для определения характеристик системы 5G по всей ее цепочке. Это стало возможным, в частности, благодаря приобретению Keysight таких компаний, как Anite и Iхіа, технологии которых дополнили наши возможности в области сетей 5G. И этот новый подход, заключающийся в предоставлении комплексных решений, - одно из ключевых отличий Keysight от наших основных конкурентов.

Какие решения сейчас в основном востребованы заказчиками, работающими в области 5G, - для сетей до 6 ГГц или выше?

Мы наблюдаем такую картину: если какаялибо компания проводит исследования в области 5С или только оценивает целесообразность таких работ, она, как правило, хочет получить систему, способную выполнять эмуляцию 
и в субшестигигагерцовом, и в более высокочастотном диапазоне. Поэтому мы разработали нашу платформу таким образом, что заказчик может начать использовать ее в диапазоне ниже 6 ГГц с расширенной полосой частот, а поз же модернизировать ее, чтобы получить возможность работы с технологиями в миллиметровом диапазоне.

У нас уже много лет существуют решения для высокочастотных измерений, которые применялись, например, в аэрокосмической отрасли. В прошлом множество таких решений было поставлено и в Россию. Сейчас эти технологии приходят в коммерческий сектор.

Когда в технике появляется принципиально новое направление, обычно какое-то время существует проблема, связанная с отсутствием соответствующего стандарта. Насколько сейчас обеспечены стандартами технологии 5G?

я бы сказал, что сейчас уже этой проблемы нет. Конечно, стандарты продолжают дорабатываться. Мы включились в процесс стандартизации 3GPP в 2015 году. Тогда технологии 5G еще только обсуждались, и Keysight содействовал разработке стандарта, внося свой вклад, как член сообщества.

\section{У нас уже много иет существуют решения дия высокочастотных измерений, которые применялись,} например, в аэрокосмической отрасли. Сейчас эти технологии приходят в коммерческий сектор

Первая версия спецификации 3GРP редакции 15, включающей первую фазу стандартизации 5G, вышла в декабре 2017 года, и с тех пор каждые три месяца в нее вносились достаточно существен ные изменения. Мы ожидаем, что окончательная версия редакции 15 должна появиться к июню 2019 года. Это достаточно полная спецификация, и отрасль очень усердно работает над тем, чтобы закончить данный процесс как можно скорее, поскольку это позволит обеспечить уверенность в том, что под развертываемые сети 5G можно будет выпускать устройства, которые будут работать с ожидаемыми характеристиками.
Этому процессу помогает то, что в мире ведутся пробные испытания сетей 5G, и результаты этих испытаний учитываются в разрабатываемом стандарте, что приближает завершение работы над ним.

Учитывает ли этот стандарт особенности работы устройств Интернета вещей в сетях 5G?

Да, узкополосные протоколы ІоТ включены в редакцию 15 спецификации 3GPP. Они существовали еще во времена 4G. Отличие в сетях 5G заключается в том, что сеть может гибко разделяться на сегменты с точки зрения производительности, использования вычислительных и коммуникационных ресурсов. Поэтому теперь можно будет определять уровень гарантированных характеристик для каждой задачи, что было очень сложно сделать в традиционных «плоских" сетях 4G.

Это создает основу для новой бизнес-модели операторов, поэтому ряд операторов проявляет большую заинтересованность в реализации Интернета вещей, в том числе в отношении критических задач, для которых требуется, чтобы задержка в сети не превышала определенной величины. Теперь операторы смогут гарантировать заданную величину задержки, объем хранилища данных, доступность вычислительных ресурсов и т. п., так что пользователь не будет испытывать тех проблем, которые существовали в традиционных сетях. Эта возможность появится, но, я думаю, появится со временем, потому что бизнес-модели для сетей 5G пока еще только формируются.

Насколько сейчас готовы технологии для создания абонентских устройств для сетей 5G?

Мы взаимодействуем с рядом крупных производителей мобильных телефонов и уже демонстрировали работу наших контрольно-измерительных приборов с их изделиями. В этой области существуют технологии, которые могут позволить создать телефоны для 5G в достаточно короткие сроки, но такие телефоны не появятся, пока не появится инфраструктура. Это можно сравнить со строительством дорог. Если вы прокладываете широкое шоссе, по нему поедут машины. Но если шоссе будет слишком узким, никому не захочется застрять в пробке, и желающих воспользоваться им будет мало. Поэтому, я думаю, сначала должны быть проложены каналы коммуникаций, создана сеть, и тогда уже под эту сеть начнут создаваться мобильные телефоны. 
здесь также стоит отметить, что за современным мобильным телефоном - смартфоном - стоят программные приложения, и в эту сторону сейчас внимательно смотрят операторы. Если они хотят получать больше прибыли, они должны входить в новый бизнес, предоставлять пользователям больше программного обеспечения.

Какие новые требования предъявляются к контрольно-измерительному оборудованию со стороны технологий 5G? Что в нем меняется?

я бы сказал, что в нем меняется почти всё.

Прежде всего, 5G - это переход к более высоким частотам. Например, Китай уже, по существу, достиг частоты 3,5 ГГц в коммуникационных системах, а сейчас они пробуют технологии 5G в диапазоне 4 ГГц. Федеральная комиссия по связи США (FCC) открыла диапазон 7,2 ГГц для сетей 5G, а это уже технологии за пределами условной границы 6 ГГц. Движение в сторону применения всё более высоких частот очевидно, поскольку это позволяет использовать более широкий частотный спектр. Соответственно, измерительное оборудование для 5G должно быть способно работать с бо́льшими частотами, чем для телекоммуникационных систем предыдущего поколения.

Второе существенное изменение связано с тем, что в 5G используются более широкие полосы частот и более сложные модуляции. Традиционное современное оборудование может выполнять модуляцию и демодуляцию сигналов с полосой порядка 160 МГц. В отношении технологий 5 м мы уже говорим о ширине полосы 800 МГц, а в некоторых случаях - 1,4 ГГц. Более широкая полоса означает то, что измерительная система должна захватывать и обрабатывать в реальном времени гораздо бо́льшие объемы данных. Поэтому и модули захвата данных, и АЦП, и устройства обработки, и архитектура измерительных приборов в целом должны обеспечивать намного более высокое быстродействие. А это значит, что изменения касаются практически всех аспектов контрольно-измерительных систем.

Кроме того, когда мы говорим о 5G, часто представляем себе в первую очередь беспроводную сеть, однако нельзя забывать, что телекоммуникационная инфраструктура включает в себя и проводные каналы, в частности связывающие базовые станции между собой и с дата-центрами.
И поскольку повышение производительности сети, ее пропускной способности, как я уже говорил, должно обеспечиваться по всей цепочке от абонентского устройства до дата-центра, проводные каналы также должны отвечать новым требованиям. На практике это означает широкое использование оптоволоконных каналов, и контрольно-измерительные средства должны позволять работать с такими каналами, выполнять демодуляцию оптических сигналов, проверку качества передачи данных и ее соответствие стандартам.

\section{Infiniium UXR - это полноценное платформенное решение, которое не только выполняет традиционные дия осциллографа задачи, но и позволяет анализировать характеристики передачи данных по оптическим каналам, а также проводить исследования в милииметровом диапазоне длин волн}

На выставке electronica 2018, прошедшей в Мюнхене в ноябре прошлого года, компания Keysight демонстрировала новые осциллографы серии Infiniium UXR, способные работать с оптическими каналами. Это решение уже готово к тем задачам, о которых вы говорите?

Да, мы очень гордимся этим решением. Я даже не называл бы его просто осциллографом. Это полноценное платформенное решение, которое не только выполняет традиционные для осциллографа задачи, но и позволяет анализировать характеристики передачи данных по оптическим каналам с пропускной способностью до 1 Тбит / с, а также проводить исследования в миллиметровом диапазоне длин волн.

Это на данный момент единственная в мире система с полосой пропускания 110 ГГц, имеющая четыре канала, которые способны независимо захватывать до 256 Гвыб./с. Для данной платформы наша компания разработала ряд новых интегральных схем, оптимизированных для решения таких задач, а также соответствующее ПО. Ведь при работе с такими скоростями необходимо очень быстро выполнять демодуляцию 
сигнала и отображать результаты в реальном времени, потому что невозможно просто сохранять такое количество захваченных необработанных данных - это потребовало бы слишком больших объемов памяти.

И для того, чтобы пользователи могли работать с оптоволоконными каналами, наша компания разработала для этой платформы оптический входной тракт, который может быть приобретен в качестве опции.

При тестировании и калибровке абонентских устройств 5G существуют определенные проблемы, связанные с необходимостью выполнения измерений беспроводным способом over-the-air. Что предлагает Кeysight для решения этих задач?

Да, такие проблемы существуют, но они относятся к высокочастотным диапазонам в терминах 5G. При работе с частотами ниже 6 ГГц измерения могут выполняться традиционно, путем подключения испытуемого устройства к измерительной аппаратуре через коаксиальный кабель.

Если же вы двигаетесь в диапазоны 28, 44 ГГц и более, то использование кабеля становится невозможным, и вам потребуется беспроводная система. И здесь существует несколько решений. Например, вы можете построить огромную камеру и очень дорогую измерительную систему, чтобы выполнять измерения в дальней зоне. Может быть, кто-то так и поступает, но этот вариант связан с очень большими затратами .

\section{Используя решения CATR, наши} заказчики могут выполнять беспроводные (over-the-air) измерения при относительно низких затратах и при этом быть уверены в высокой точности результатов измерений

To, что предлагаем мы,- концепция CATR (Compact Antenna Test Range - измерение параметров антенн на малом расстоянии). В этом решении применяется преобразование параметров ближней зоны в параметры дальней. Мы провели ряд исследований, благодаря которым нам удалось достичь очень хорошего уровня точности измерений при достаточно низкой стоимости решения. Кроме того, следуя принципу предоставлять заказчикам законченные решения, мы выполнили очень плотную интеграцию этой системы и наших программных средств. Так что теперь наши заказчики могут выполнять беспроводные измерения при относительно низких затратах и при этом быть уверены в высокой точности результатов измерений.

Контроль и измерения в области телекоммуникационного оборудования не ограничиваются каналами передачи данных. Необходимо контролировать и параметры электропитания, и другие характеристики. Как построено взаимодействие между подразделениями Keysight, чтобы заказчики могли воспользоваться для решения своих задач предложениями компании из смежных областей?

Это очень хороший вопрос. Как я уже говорил, Keysight Technologies позиционирует себя как поставщик комплексных решений, а не отдельных приборов. Именно такие решения сейчас нужны заказчикам. Мы это видим по той обратной связи, которую получаем от наших подразделений по всему миру, в том числе и из России. И в том, чтобы удовлетворить эту потребность, мы видим одну из основных наших задач.

Поэтому некоторое время назад руководством компании Keysight было принято решение изменить ее структуру. Если раньше мы были организованы по продуктовому принципу, то теперь наша структура выстроена на основе подразделений, каждое из которых сосредоточено на определенном сегменте промышленности. Иными словами, у нас теперь нет подразделения, которое продает, например, именно анализаторы спектра, но каждое из подразделений может поставить заказчику из своего сегмента такой прибор, если он ему нужен для решения его задач.

я отвечаю за подразделение решений для телекоммуникаций. Это одно из крупнейших направлений Keysight, на которое приходится около половины оборота компании - порядка 2,5 млрд долл. В рамках этого большого подразделения есть три бизнес-единицы. Одна отвечает за сегмент разработки и производства устройств для коммуникации, другая - за сегмент телекоммуникационной инфраструктуры, что включает в себя базовые станции, технологии проводных каналов передачи данных и т. п. И третья бизнес-единица работает с аэрокосмическим и оборонным сектором. Это очень важное направление, поскольку многие годы самые 
сложные и передовые технологии появлялись именно в этом секторе

Отмечу, что это изменение структуры было выполнено в период, который никак нельзя назвать сложным для компании. Обычно компании реорганизуются, когда у них возникают финансовые сложности, но у нас всё было по-другому. Мы это сделали не для того, чтобы выйти из тяжелой ситуации, решить собственные проблемы, а для того, чтобы стать еще лучше для наших заказчиков, помочь им двигаться вперед быстрее.

Таким образом, в Keysight есть команды, которые сосредоточены на определенных клиентах, на их потребностях. А за этим стоит то, что мы называем центрами передовых технологий (centers of excellence). Каждый центр обладает опытом и знаниями в определенной области. В них разрабатываются новые технологии и продукты. И когда у того или иного подразделения возникает задача дополнить решение для своего заказчика новым изделием или технологией из конкретной технической области, оно обращается в соответствующий центр передовых технологий. Таким образом, заказчики из любого сектора промышленности могут получить комплексное решение, включающее все необходимые технологии из разных технических областей.

Такая организация компании является существенной составляющей нашего успеха. Достижения Keysight за последний год во многом определяются именно этим.

Компания Keysight - глобальная и наверняка имеет несколько производств, расположенных в разных частях мира. Как обеспечивается единство результатов измерений, выполняемых с помощью приборов Keysight, которые были произведены на разных производственных площадках?

я знаю, что такая проблема существует у некоторых наших конкурентов, но у нас она решена. На двух осциллографах Keysight, изготовленных в разных странах, вы получите результаты, совпадающие с очень высокой точностью. Наш секрет - не только в передовых аппаратных средствах, но и в том, как у нас организованы процессы производства, контроль в рамках техпроцессов и т. п. Поэтому, где бы ни были произведены те или иные наши приборы, вы можете быть уверены, что они изготовлены по одинаковым технологиям, в них применяется одинаковое пО, они прошли одинаковый контроль, калибровку - всё в соответствии с нашими процедурами, отвечающими самым высоким стандартам метрологии. Мы так работаем уже много лет, и этот подход хорошо зарекомендовал себя.

И, конечно, мы обеспечиваем прослеживаемость калибровки наших приборов до эталонов Национального института стандартов и технологий США (NIST).

\section{Новая структура Keysight на основе подразделений, каждое из которых сосредоточено на определенном сегменте промышиенности, позволяет заказчикам получать комплексные решения, вкиючающие все необходимые технологии из разных технических областей}

В России действуют свои национальные метрологические стандарты. Как обеспечивается соответствие решений Keysight российским требованиям?

Конечно, в каждой стране есть свои особенности, свои требования. Мы всегда стремимся создавать наши решения таким образом, чтобы они были применимы по всему миру, но в некоторых случаях требуется определенная доработка, модификация под конкретный рынок. и, когда это необходимо, мы выполняем такую кастомизацию.

У нас в разных странах есть команды, которые помогают нам лучше отвечать требованиям локальных рынков, понимать, что нужно заказчикам именно в этой стране. Такая команда есть у нас и в России. Она не только содействует лучшему взаимопониманию между Keysight и российскими компаниями, но также берет на себя задачи по выполнению процедур, которые требуются в соответствии с нормативными требованиями, действующими в Российской Федерации. Так, наши приборы оперативно заносятся в Государственный реестр средств измерений рФ и получают необходимые сертификаты, чтобы российские заказчики могли воспользоваться нашими новейшими решениями для создания передовой конкурентоспособной продукции

\section{Спасибо за интересный рассказ.}

С С. Данасекараном беседовал Ю. Ковалевский 